\title{
Identification of Key Genes and Pathways associated with Endometriosis by Weighted Gene Co-expression Network Analysis
}

\author{
Jingni $\mathrm{Wu}^{1,2}$, Xiaoling Fang 1 , Xiaomeng $\mathrm{Xia}^{1 凶}$ \\ 1. Department of Obstetrics and Gynecology, The Second Xiangya Hospital, Central South University, Changsha, Hunan 410011, China \\ 2. Department of Obstetrics and Gynecology, David Geffen School of Medicine, University of California, Los Angeles, California 90095, United States. \\ $\triangle$ Corresponding author: Xiaomeng Xia, E-mail: xiaxiaomeng@csu.edu.cn.
}

(C) The author(s). This is an open access article distributed under the terms of the Creative Commons Attribution License (https://creativecommons.org/licenses/by/4.0/). See http://ivyspring.com/terms for full terms and conditions.

Received: 2021.06.04; Accepted: 2021.07.26; Published: 2021.08.03

\begin{abstract}
Background: Endometriosis is a common gynecological disorder with high rates of infertility and pelvic pain. However, its pathogenesis and diagnostic biomarkers remain unclear. This study aimed to elucidate potential hub genes and key pathways associated with endometriosis in ectopic endometrium (EC) and eutopic endometrium (EU).

Material and Method: EC and EU-associated microarray datasets were obtained from the gene expression omnibus (GEO) database. Gene set enrichment analysis was performed to obtain further biological insight into the EU and EC-associated genes. Weighted gene co-expression network analysis (WGCNA) was performed to find clinically significant modules of highly-correlated genes. The hub genes that belong to both the weighted gene co-expression network and protein-protein interaction (PPI) network were identified using a Venn diagram.

Results: We obtained EC and EU-associated microarray datasets GSE7305 and GSE120103. Genes in the EC were mainly enriched in the immune response and immune cell trafficking, and genes in the EU were mainly enriched in stress response and steroid hormone biosynthesis. PPI networks and weighted gene co-expression networks were constructed. An EC-associated blue module and an EU-associated magenta module were identified, and their function annotations revealed that hormone receptor signaling or inflammatory microenvironments may promote EU passing through the oviducts and migrating to the ovarian surfaces, and adhesion and immune correlated genes may induce the successful ectopic implantation of the endometrium (EC). Twelve hub genes in the EC and sixteen hub genes in the EU were recognized and further validated in independent datasets.

Conclusion: Our study identified, for the first time, the hub genes and enrichment pathways in the EC and EU using WGCNA, which may provide a comprehensive understanding of the pathogenesis of endometriosis and have important clinical implications for the treatment and diagnosis of endometriosis.
\end{abstract}

Key words: endometriosis; WGCNA; biological markers; pathway

\section{Introduction}

Endometriosis is an estrogen-dependent gynecological disorder characterized by the growth of endometrium in ectopic locations [1]. A total of $30-50 \%$ of women with endometriosis suffer from pain and/or unexplained infertility [2]. Despite several theories (i.e., retrograde menstruation, coelomic metaplasia, Müllerian remnants) that have been proposed, the pathogenesis of endometriosis is still unknown. The widely accepted retrograde menstrual reflux hypothesis states that eutopic endometrium (EU) migrates and survives outside the cavity of uterus, then establishes new endometriosis lesions. It is believed that the elucidation of molecular and functional specificities of the ectopic endometrium (EC) and EU facilitates a better understanding of the complex physiopathology of endometriosis. Previous studies have shown that the EC may behave differently from its eutopic counterpart [3]. However, these studies mainly focused on a few molecules or the gene expression 
differences between different tissues, without considering the intrinsic relationship between these genes. In addition, there is still room for improvement of the bioinformatics algorithm in analyzing these transcriptomic data, and the specific biomarkers and roles of the EC and EU in endometriosis remain uncertain. Therefore, our study for the first time explored the genomic alteration profiles of the two entities of endometriosis using weighted gene co-expression network analysis (WGCNA) to identify endometriosis-associated biomarkers and pathways.

Currently, there is no standard protocol for analyzing transcriptomic data. Network analysis is a promising direction that allows for a greater ability to recognize biological themes or pathways. It combines biology and network science to study the relationships of interacting components, which may provide novel and comprehensive insights into the diseases from the level of multiple genes [4]. WGCNA is a network method for identifying highly correlated gene expression modules in different samples and analyzing the correlation between the module and disease type/clinical phenotype. Hence, WGCNA has been widely used to explore the biomarkers and therapeutic targets of various diseases, such as breast cancer [5]. WGCNA has also been used to identify biologically related modules. For example, Wang et al. found fifteen hub genes that were highly correlated with the progression and prognosis of clear cell renal cell carcinoma using WGCNA [6]. As a result, using WGCNA, we attempted to identify the modules of co-expressed genes highly associated with endometriosis and their key drivers. Meanwhile, we tried to explore the key pathways of the EC and EU in the pathogenesis of endometriosis. Our study may provide a better understanding of the disorder through the comparison between the eutopic and ectopic endometrium, and provide a new insight into the molecular mechanisms underlying the pathogenesis of endometriosis.

\section{Materials and methods}

\section{Study design}

To illustrate the data preprocessing, analysis and validation, a schematic flow diagram of the study is presented in Figure 1.

\section{Data acquisition}

Expression profiles of endometriosis-associated mRNAs in GSE7305, GSE120103, GSE7307 and GSE51981 were downloaded from Gene Expression Omnibus (GEO) database. The microarray datasets GSE7305 and GSE120103 with complete clinical information and same menstrual cycle were used as training sets to identify hub differentially expressed genes (DEGs) of endometriosis, GSE7307 and GSE51981 were used as test sets to validate our results, respectively. Dataset GSE7305 [7] performed on the GPL570 platform (Affymetrix Human Genome U133 Plus 2.0 Array) was used to recognize hub DEGs in ovarian endometrioma, which includes 10 ovarian endometriomas from women with endometriosis (EC) and 10 normal endometria (Ctrl). Dataset GSE120103 [8] performed on the GPL6480 platform (Agilent014850 Whole Human Genome Microarray 4x44K G4112F) was applied to identify hub DEGs in eutopic endometrium, which includes 9 eutopic endometria from fertile women with endometriosis (EU) and 9 Ctrl. Dataset GSE7307 performed on the GPL570

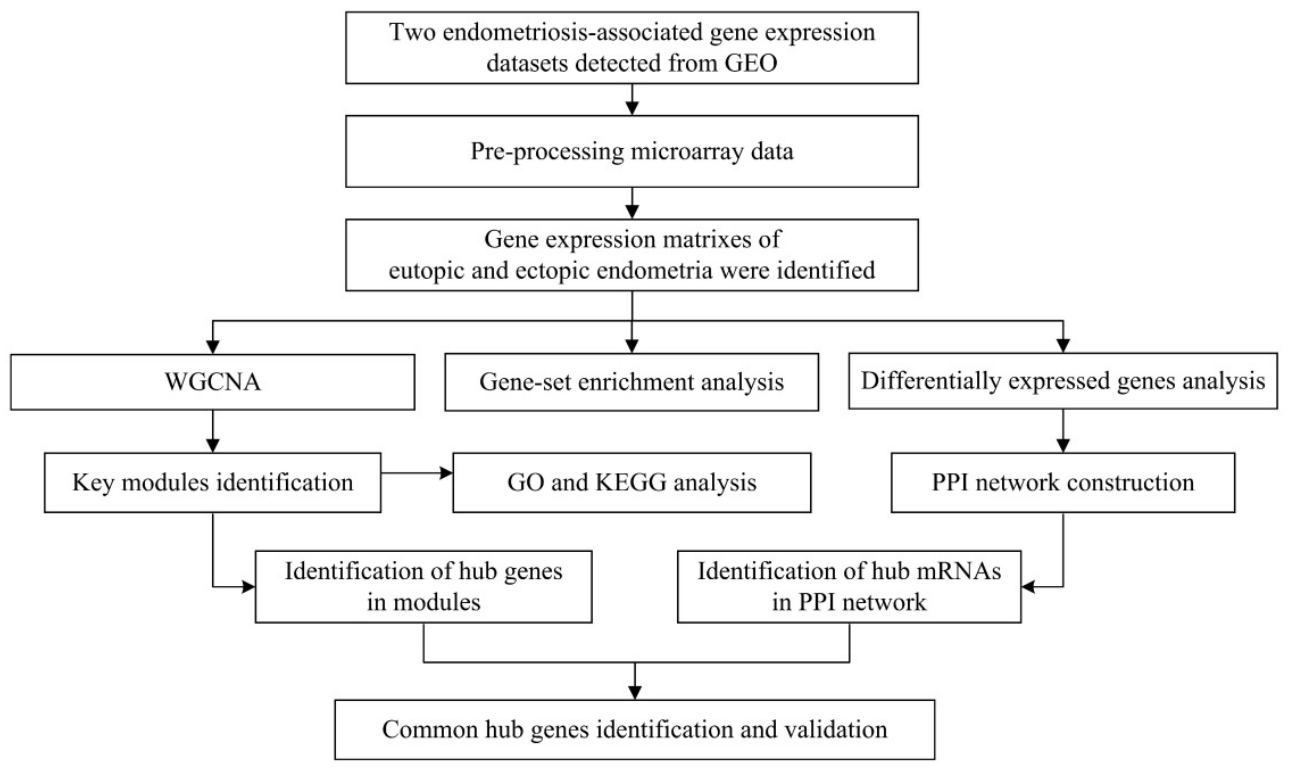

Figure 1. Flow diagram of strategy for data preparation, preprocessing, and analysis. 
platform (Affymetrix Human Genome U133 Plus 2.0 Array) was used to validate EC-associated hub DEGs, which includes $23 \mathrm{EC}$ and $18 \mathrm{Ctrl}$. And Dataset GSE51981 performed on the GPL570 platform (Affymetrix Human Genome U133 Plus 2.0 Array) was used to validate EC-associated hub DEGs, which includes $38 \mathrm{EU}$ and $71 \mathrm{Ctrl}$.

\section{Data preprocessing and differentially expressed genes (DEGs) identification}

Limma (linear models for microarray data) package $[9,10]$ in $R$ software was utilized to correct the data background and identify the DEGs in EC vs Ctrl and EU vs Ctrl groups. Batch effect was removed using the limma package removeBatchEffect function. Benjamin and Hochberg method was used for multiple testing corrections [11]. The false discovery rate (FDR) $<0.05$ and $\mid \log _{2}$ (Fold Change) $\mid\left(\left|\log _{2} \mathrm{FC}\right|\right)$ $\geq 1$ was the cut-off criteria for screening DEGs.

\section{Gene-set enrichment analysis (GSEA)}

To evaluate the molecular mechanisms of endometriosis, GSEA of the gene expression profiles of GSE7305 and GSE120103 was performed using the "ClusterProfiler" package in R (http://www. bioconductor.org/packages/release/bioc/html/ clusterProfiler.html). The genes were listed based on their expression levels, and were further mapped to the annotated gene sets of c5 (Gene Ontology (GO) gene sets) and c2 (curated gene sets) in Molecular Signatures Database (MSigDB). Gene sets with P-value $<0.05$ and FDR $<25 \%$ are considered as significant [12].

\section{Protein-protein interaction (PPI) network construction}

The search tool for retrieval of interacting genes (STRING) database was used to identify the interactions among DEGs with the parameters of protein interaction score $>0.4$. Thereafter, the PPI network is constructed by Cytoscape. The potential hub DEGs were determined by Molecular Complex Detection (MCODE) plug-in (K-score >3) [13].

\section{Weighted gene co-expression network construction}

We used the WGCNA R package to establish co-expression networks [14] for the genes in GSE7305 and GSE120103. The unqualified genes were screened out. A matrix of genes' similarity by Pearson's correlation analysis was created. Appropriate soft threshold power $(\beta)$ was applied to strengthen this matrix to a scale-free co-expression network. For this purpose, we choose the lowest power (14 or 22) for which the scale-free topology fit index curve flattens out upon reaching a high value (above 0.8).
Furthermore, the adjacency matrix was transformed into the topological overlap matrix (TOM). Genes with higher TOM values indicate higher connectivities in the network; that is, more adjacencies to other network-generated genes $[15,16]$. Meanwhile, genes were clustered hierarchically by the TOM-based dissimilarity (1-TOM) measure. The highly correlated genes were assigned to the same module.

\section{Clinically significant module identification and function analysis}

The correlation between modules and clinical traits was investigated by the module-trait relationship analysis of WGCNA. The modules that most relevant to the clinical traits could be identified. In this study, the endometriosis-associated blue and magenta modules were chosen for the subsequent analyses. Metascape was used to explore the function annotations (GO biological processes and Kyoto Encyclopedia of Genes and Genomes (KEGG) pathways) of these two modules. Terms with $P$-value $<0.01$, count $\geq 3$ and an enrichment factor $>1.5$ were considered statistically significant.

\section{Hub genes identification and verification}

We analyzed the gene significance (GS, the correlation between the gene and a clinical phenotype of interest) and module membership (MM, the correlation between gene expression profile and module eigengene) of each gene in the clinically significant blue and magenta modules. The module eigengene is defined as the main component of the module's gene expression matrix. $|\mathrm{MM}|>0.6$ and $|G S|>0.8$ were set as the threshold for screening candidate hub genes that strongly associated with EC or EU. In the end, the Venn diagram was performed to identify the common hub genes from PPI network analysis and WGCNA. In addition, GSE7307 and GSE51981 were used as validation data sets. "ggplot2" (Ito \& Murphy, 2013) R package was applied to show relative expression of the identified hub genes in different comparison groups (EC vs Ctrl or EU vs Ctrl).

\section{Results}

\section{Identification of differentially expressed genes}

With the cut-off criteria $($ FDR $<0.05$ and $\left|\log _{2} \mathrm{FC}\right|>1$ ), a total of 1487 DEGs (824 upregulated and 663 downregulated) were identified between the EC and Ctrl in the GSE7305 dataset, and a total of 5794 DEGs (1974 upregulated and 3820 downregulated) were identified between the EU and Ctrl in the GSE120103 dataset. Volcano plots show the variation of DEGs in the EC versus Ctrl (Figure 2A) and EU versus Ctrl (Figure 2B). 
A

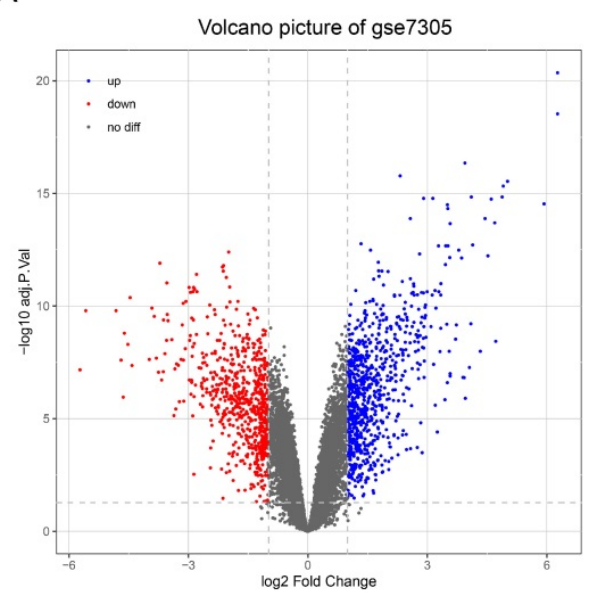

B

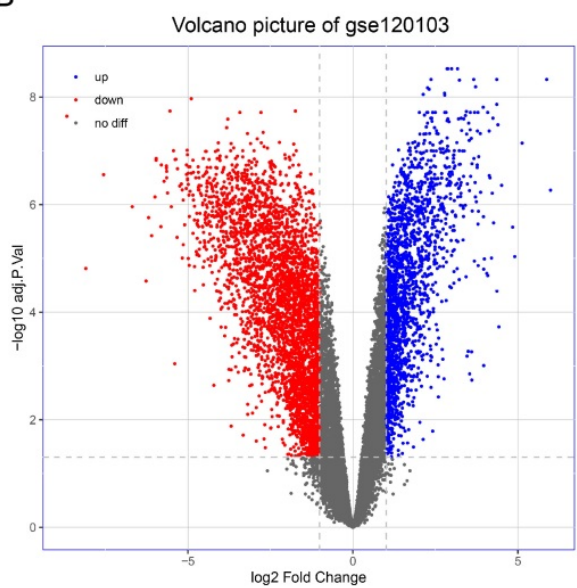

Figure 2. Differentially expressed genes in endometriosis. (A) Volcano map of differentially expressed genes in EC compared with Ctrl. (B) Volcano map of differentially expressed genes in EU compared with Ctrl. EC: ectopic endometrium from patient with endometriosis. EU: eutopic endometria from patient with endometriosis. Ctrl: endometrium from patient without endometriosis.

A
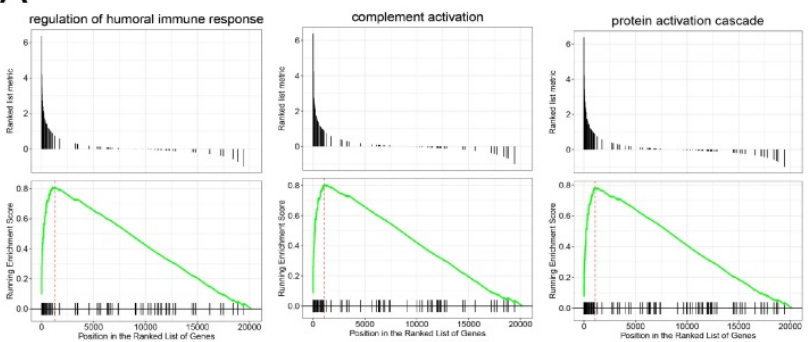

C
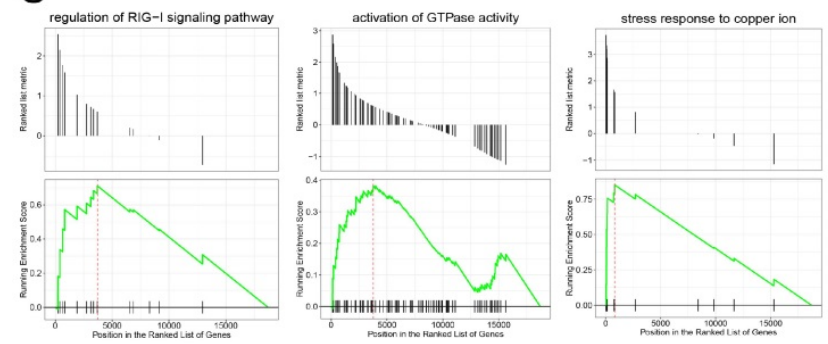

B
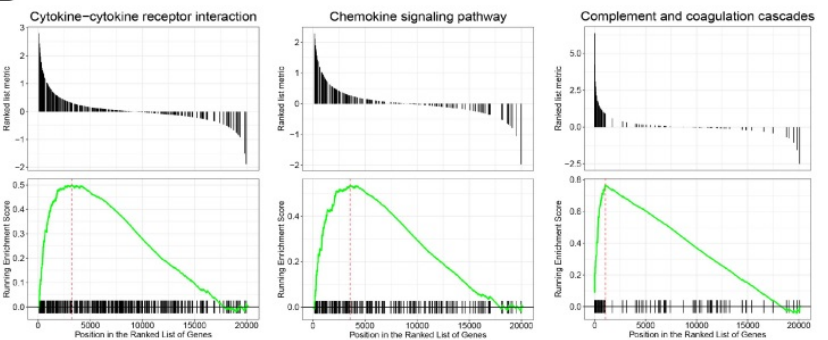

D
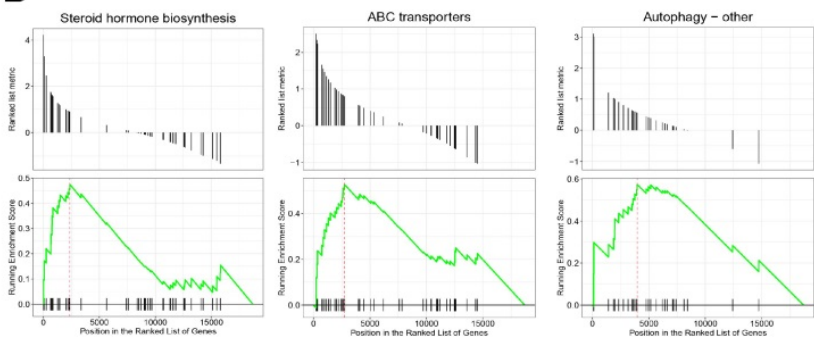

Figure 3. Gene set enrichment analysis (GSEA) of genes in endometriosis. GSEA-identified biological processes (A) and pathways (B) with significant enrichment in EC compared with Ctrl. GSEA-identified biological processes (C) and pathways (D) with significant enrichment in EU compared with Ctrl.

\section{Gene-set enrichment analysis}

GSEA revealed the genes in the EC were mainly enriched in the immune response and immune cell trafficking, such as protein activation cascade, complement activation, regulation of humoral immune response, complement and coagulation cascades pathway and chemokine signaling pathway (Figure 3A-B), and the genes in the EU were mainly enriched in the stress response and steroid hormone biosynthesis, such as the stress response to copper ion, activation of GTP hydrolases (GTPases) activity, Human ATP-binding cassette (ABC) transporter dependent pathway and steroid hormone biosynthesis pathway (Figure 3C-D). We explored the functions of the two entities of endometriosis using genomic alteration profiles. These function annotations revealed the distinct roles of the EC and $\mathrm{EU}$ in the pathological process of endometriosis, which demonstrated the reliability of our results.

\section{Construction of protein-protein interaction network}

To identify the candidate hub genes, the most differentially expressed 362 genes in the EC versus Ctrl and 1992 genes in the EU versus Ctrl were selected for the PPI network construction. The MCODE clustering algorithm was applied to analyze the PPI network. With a threshold of k-scores $>3$, seven clusters with 78 candidate hub genes in the EC and 21 clusters with 205 candidate hub genes in the EU were selected. Figure 4A-D depicts the top two clusters in the EC and EU. 
A

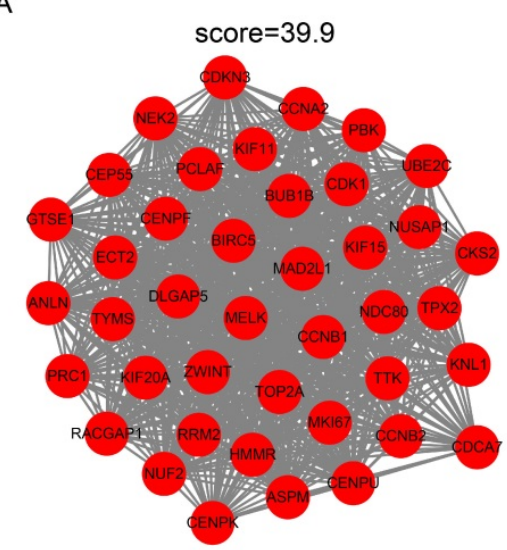

C

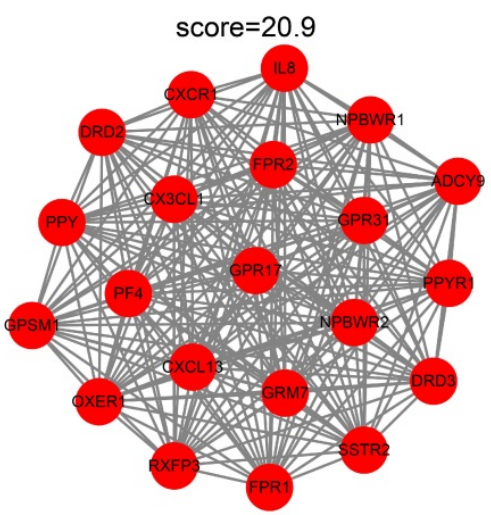

B

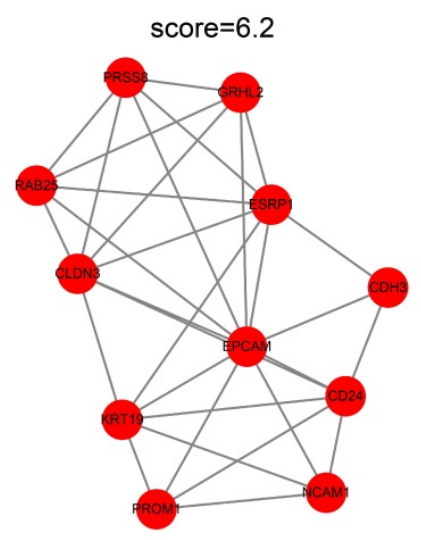

D

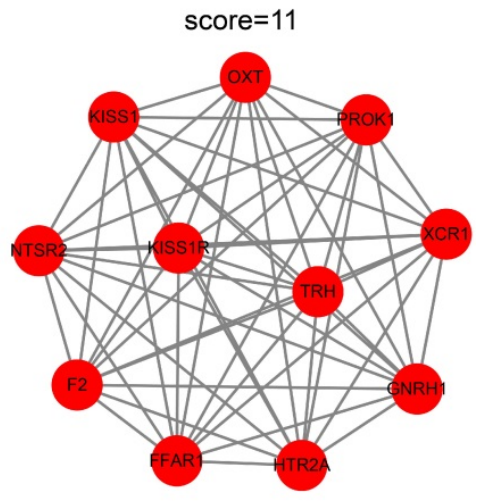

Figure 4. Protein-protein interaction network cluster analysis. The PPI network of differentially expressed genes in EC versus Ctrl or EU versus Ctrl was constructed using the STRING website. The MCODE clustering algorithm was applied to the network to identify the potential hub genes. Top two clusters in each group are shown in the figure. In the EC-associated PPI network, cluster 1 consists of 11 nodes and 31 edges (A) and cluster 2 consists of 11 nodes and 31 edges (B). In the EU-associated PPI network, cluster 1 consists of 21 nodes and 209 edges (C) and cluster 2 consists of 11 nodes and 55 edges (D). PPI: protein-protein interaction; STRING, search tool for retrieval of interacting genes/proteins.

\section{Construction of the weighted gene co- expression network and identification of clinically significant modules}

The values of $\beta=14$ (scale free $R^{2}=0.80$ ) and $\beta=$ 22 (scale free $R^{2}=0.85$ ) were selected as the soft-threshold powers to ensure scale-free networks using R package of "WGCNA" (Figure 5A-B). Genes with similar expression patterns were clustered into co-expression modules that were displayed in different colors. A total of 58 and 39 modules were identified (Figure 5C-D).

The relevance between each module and clinical information was shown in the module-trait relationship (Figure 5E-F). In this situation, we focused on the EC and EU-associated key modules. The blue module, containing 2036 genes, was most correlated with the EC $\left(\mathrm{R}=0.87, \mathrm{p}=5 \times 10^{-6}\right)$. Meanwhile, the magenta module, containing 768 genes, was most correlated with the EU $\left(R=0.97, p=1 \times 10^{-12}\right)$. Hence, the blue and magenta modules were clinically significant and used for the following analyses in this study.

\section{Function analysis of the most significant module}

To explore the function mechanism of genes in the clinically significant modules, GO analysis and KEGG analysis were conducted. Function analysis revealed that the main biological processes and pathways of the blue module were regulation of cell adhesion, autophagy, FoxO signaling pathway and focal adhesion pathway (Figure $6 \mathrm{~A}-\mathrm{B})$, and those of the magenta module were regulation of the mitogen-activated protein kinase (MAPK) cascade, regulation of the growth hormone receptor, the tumor necrosis factor (TNF) signaling pathway and NOD-like receptor signaling pathway (Figure 6C-D). These function annotations for the blue and magenta modules are listed in Table 1 and Table 2. The genes in the top EC-associated module mainly played roles in autophagy, focal adhesion and cancer, while those in the top EU-associated module were involved in creating an estrogen-rich and inflammatory microenvironment.

\section{Identification of hub genes}

Hub genes in the co-expression network are characterized by high intramodular connectivity which is measured by the value of GS and MM. In Figure 7A and 7B, the scatterplots of GS (y-axis) vs. MM (x-axis) are shown in the blue $(R=0.95$, $\left.\mathrm{p}<1 \times 10^{-200}\right)$ and magenta $\left(\mathrm{R}=0.8, \mathrm{p}<4.2 \times 10^{-172}\right)$ modules. MM was highly correlated with GS in each module, which indicated that the hub genes in the co-expression modules were highly correlated with endometriosis. With the threshold of $|\mathrm{MM}|>0.6$ and $|G S|>0.8$, using WGCNA, we identified 735 candidate hub genes in the blue module, and 329 candidate hub genes in the magenta module.

For the identification of endometriosisassociated hub genes, we compared the hub genes in the co-expression and PPI networks. We finally identified 16 overlapping hub genes in the blue module (Figure 7C) and 12 overlapping hub genes in the magenta module (Figure 7D). These 28 hub genes are listed in Table 3. 
A

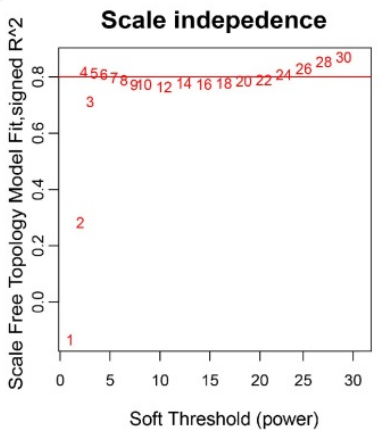

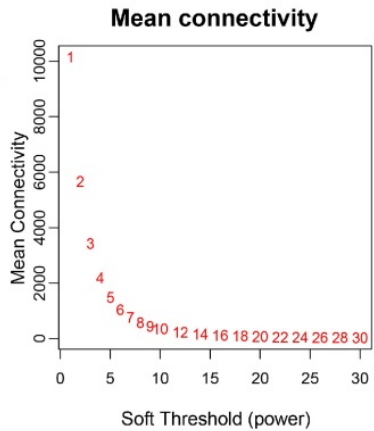

B
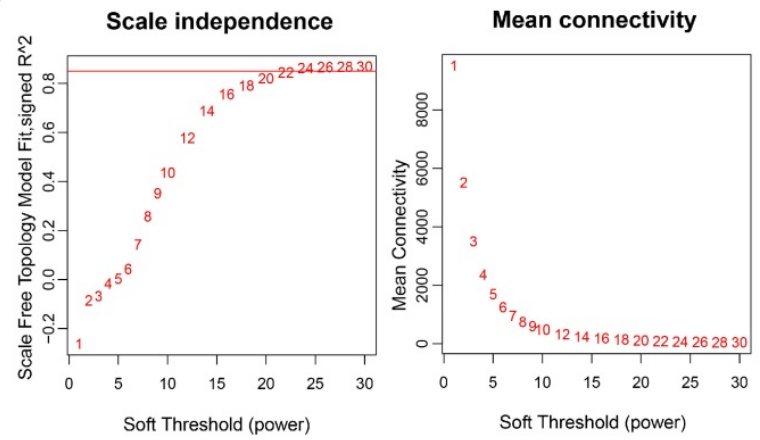

C

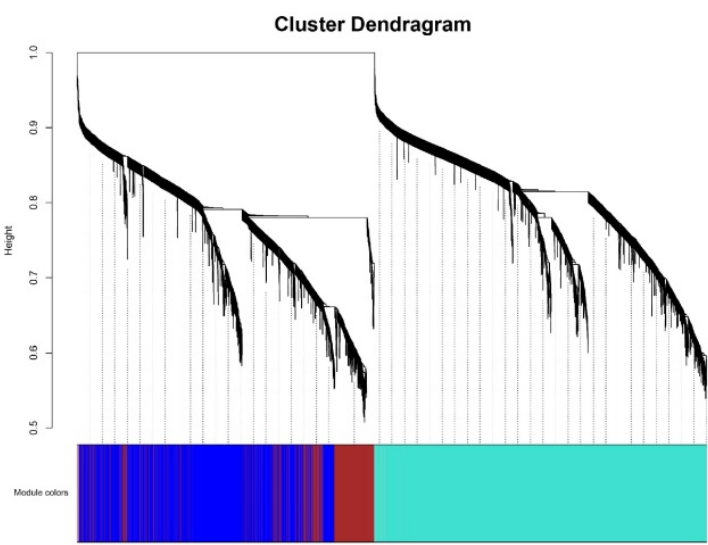

E

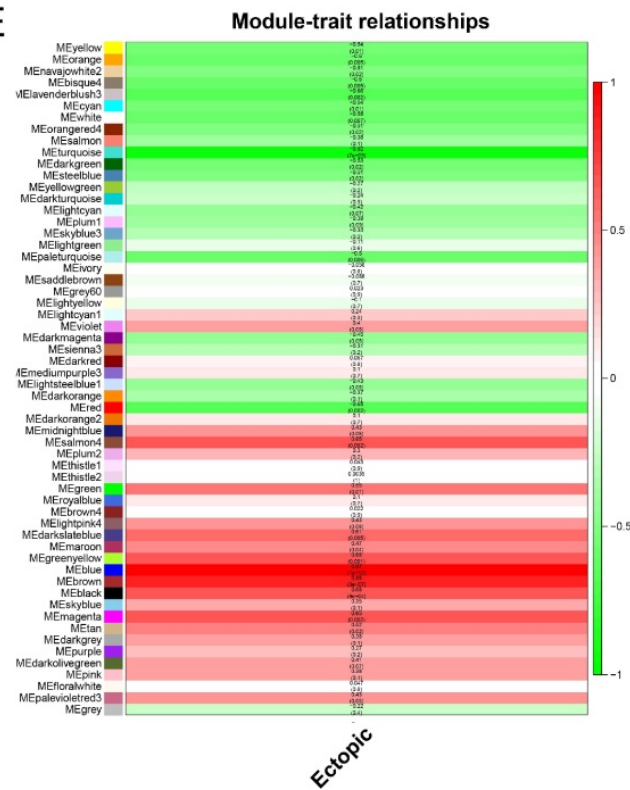

D

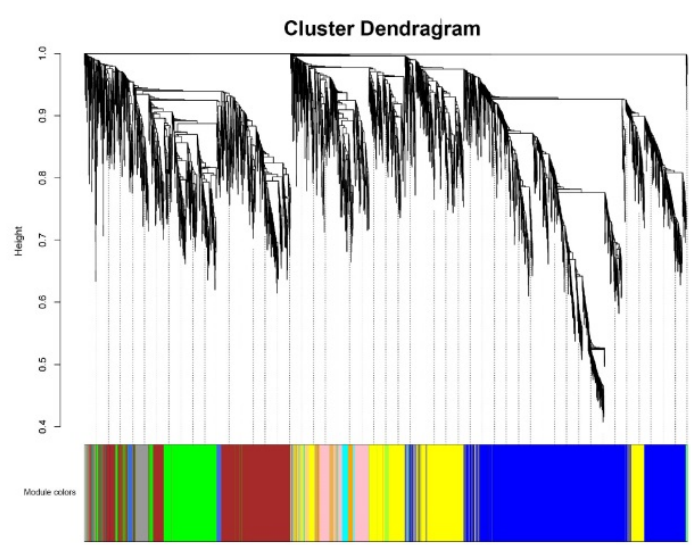

F

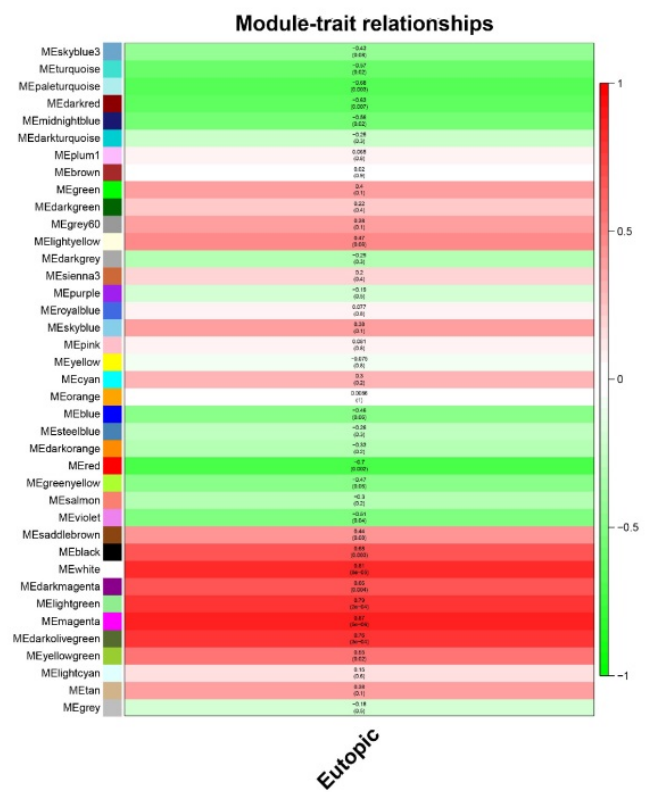

Figure 5. Weighted gene co-expression network analysis of genes in endometriosis. Analysis of the scale-free topology fit index and the mean connectivity for various soft-threshold powers $(\beta)$ for the genes in the EC (A) and EU (B). Dendrogram of all expressed genes in the EC (C) and EU (D) clustered based on a dissimilarity measure (1-TOM). Determination of module-trait relationship of the EC (E) or EU (F) group in endometriosis and identification of the most clinically relevant modules; each row indicates a module eigengene (the first principal component of the gene expression matrix in a module), and each column represents a clinical trait. TOM: topological overlap matrix.

Table 1. The GO and KEGG Pathway analysis of genes in the blue module

\begin{tabular}{|c|c|c|c|}
\hline Category & Term & Description & $\log (\mathrm{p}$-value $)$ \\
\hline GO Biological Processes & GO:0030029 & actin filament-based process & -20.593297 \\
\hline GO Biological Processes & GO:0071417 & cellular response to organonitrogen compound & -20.2406423 \\
\hline GO Biological Processes & GO:0030155 & regulation of cell adhesion & -20.0365408 \\
\hline GO Biological Processes & GO:0006914 & autophagy & -15.9477686 \\
\hline GO Biological Processes & GO:0010942 & positive regulation of cell death & -14.3658968 \\
\hline
\end{tabular}




\begin{tabular}{|c|c|c|c|}
\hline Category & Term & Description & Log(p-value) \\
\hline GO Biological Processes & GO:0007264 & small GTPase mediated signal transduction & -14.0979829 \\
\hline GO Biological Processes & GO:0070848 & response to growth factor & -13.3811234 \\
\hline GO Biological Processes & GO:0045055 & regulated exocytosis & -13.372418 \\
\hline GO Biological Processes & GO:0051345 & positive regulation of hydrolase activity & -13.0915765 \\
\hline GO Biological Processes & GO:0001568 & blood vessel development & -12.7380519 \\
\hline GO Biological Processes & GO:0000904 & cell morphogenesis involved in differentiation & -12.5393524 \\
\hline GO Biological Processes & GO:0043062 & extracellular structure organization & -12.4268848 \\
\hline GO Biological Processes & GO:0009611 & response to wounding & -12.0333641 \\
\hline GO Biological Processes & GO:0043408 & regulation of MAPK cascade & -11.5789888 \\
\hline GO Biological Processes & GO:0022604 & regulation of cell morphogenesis & -11.4691072 \\
\hline GO Biological Processes & GO:0002576 & platelet degranulation & -11.2263844 \\
\hline GO Biological Processes & GO:0019221 & cytokine-mediated signaling pathway & -10.9869128 \\
\hline GO Biological Processes & GO:0045936 & negative regulation of phosphate metabolic process & -10.9676618 \\
\hline GO Biological Processes & GO:0003012 & muscle system process & -10.2900458 \\
\hline GO Biological Processes & GO:0051129 & negative regulation of cellular component organization & -10.2118548 \\
\hline KEGG Pathway & hsa04068 & FoxO signaling pathway & -9.5 \\
\hline KEGG Pathway & hsa04510 & Focal adhesion & -9 \\
\hline KEGG Pathway & hsa04014 & Ras signaling pathway & -8.6 \\
\hline KEGG Pathway & hsa04010 & MAPK signaling pathway & -8.2 \\
\hline KEGG Pathway & hsa05200 & Pathways in cancer & -7.3 \\
\hline KEGG Pathway & hsa05205 & Proteoglycans in cancer & -7.2 \\
\hline KEGG Pathway & hsa04380 & Osteoclast differentiation & -6.8 \\
\hline KEGG Pathway & hsa04610 & Complement and coagulation cascades & -6.6 \\
\hline KEGG Pathway & hsa04630 & Jak-STAT signaling pathway & -6 \\
\hline KEGG Pathway & hsa05418 & Fluid shear stress and atherosclerosis & -5.9 \\
\hline KEGG Pathway & hsa04270 & Vascular smooth muscle contraction & -5.9 \\
\hline KEGG Pathway & hsa04144 & Endocytosis & -5.9 \\
\hline KEGG Pathway & hsa04727 & GABAergic synapse & -5.8 \\
\hline KEGG Pathway & hsa04137 & Mitophagy - animal & -5.5 \\
\hline KEGG Pathway & hsa04915 & Estrogen signaling pathway & -5.5 \\
\hline KEGG Pathway & hsa04621 & NOD-like receptor signaling pathway & -5.1 \\
\hline KEGG Pathway & hsa05202 & Transcriptional misregulation in cancer & -4.2 \\
\hline KEGG Pathway & hsa04720 & Long-term potentiation & -4.1 \\
\hline KEGG Pathway & hsa04668 & TNF signaling pathway & -3.8 \\
\hline KEGG Pathway & hsa04020 & Calcium signaling pathway & -3.7 \\
\hline
\end{tabular}

Note: GO Biological Processes: Gene ontology analysis of biological process. KEGG: Kyoto Encyclopedia of Genes.

A

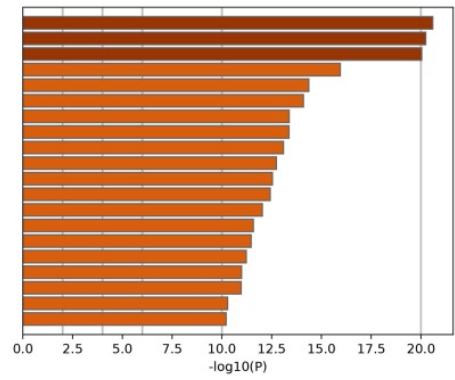

B

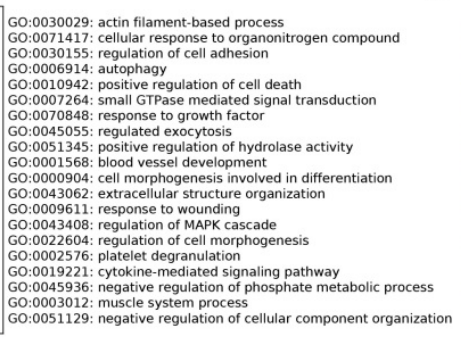

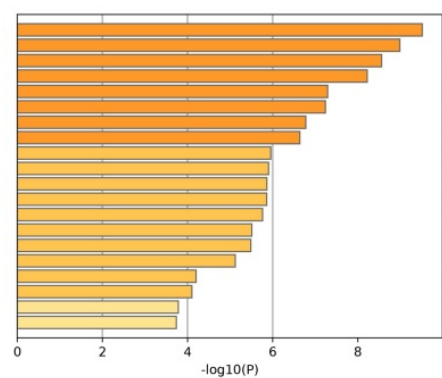

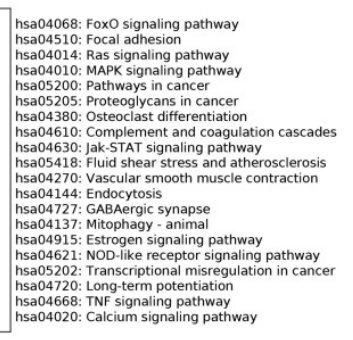

C

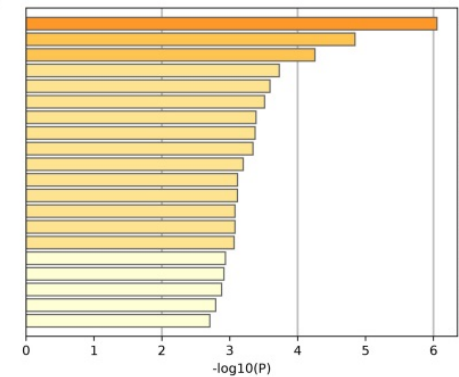

D

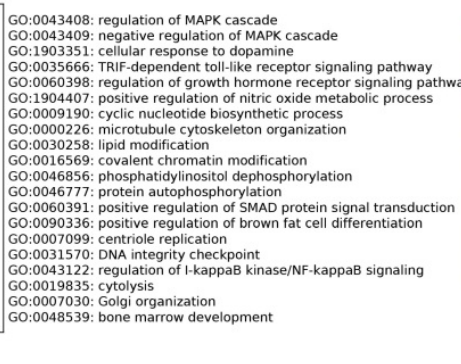

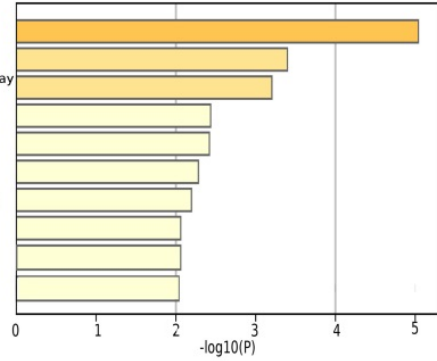

hsa00310: Lysine degradation hs504668: TNF signaling pathway. hso04621: N0D-like receptor signaling pathway hsa05020: Prion diseases hs:00514: Other types of 0 -glycan biosynthesis hso04140: Autophagy - animal hsa04912: GnRH signaling pathway hs504070: Phosphatidylinositol signaling system hsa04550: Apoptosis hsa04550: Signaling pathways regulating

Figure 6. Function annotations of clinically significant module. The GO biological processes (A) and KEGG pathways (B) of genes in EC-associated blue module. The $\mathrm{GO}$ biological process (C) and KEGG pathway (D) of genes in EU-associated magenta module. GO: Gene Ontology; KEGG: Kyoto Encyclopedia of Genes and Genomes. 
A

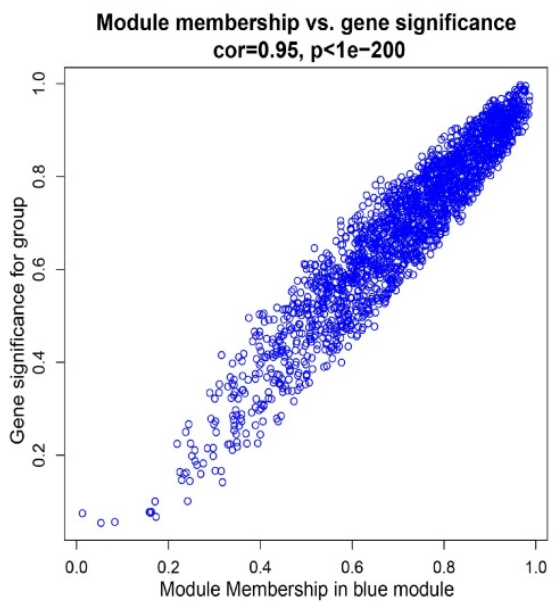

C

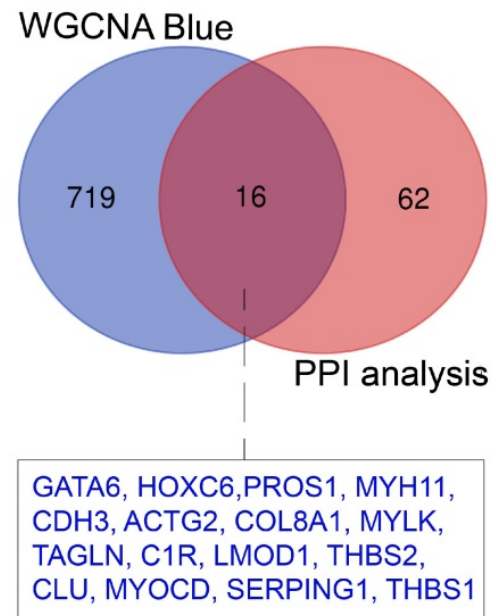

B

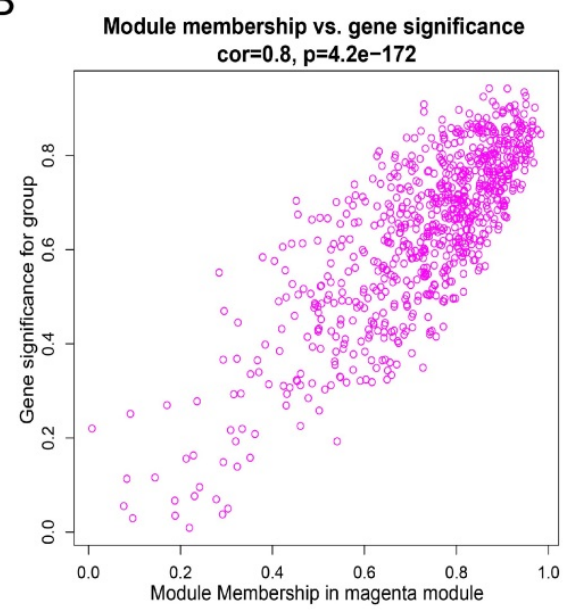

D
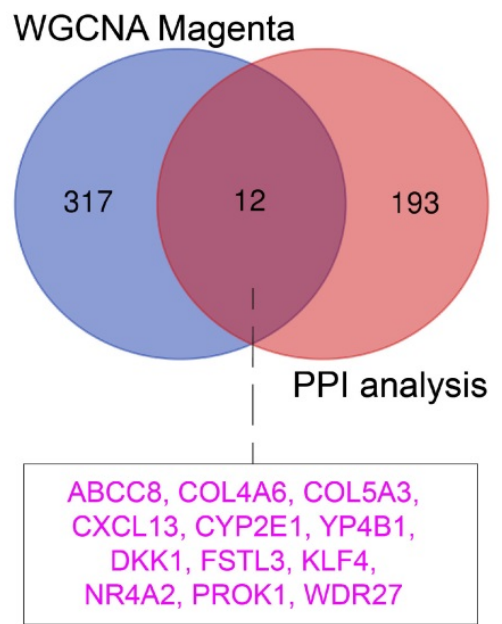

Figure 7. Identification of hub genes in endometriosis. (A) Scatterplot of module eigengenes related to EC in the blue co-expression module. (B) Scatterplot of module eigengenes related to EU in the magenta co-expression module. (C) Venn plot of common hub genes in blue module and PPI network. (D) Venn plot of common hub genes in magenta module and PPI network.

Table 2. The GO and KEGG Pathway analysis of genes in the magenta module

\begin{tabular}{|c|c|c|c|}
\hline Category & Term & Description & $\log (\mathrm{p}$-value $)$ \\
\hline GO Biological Processes & GO:0043408 & regulation of MAPK cascade & -6.05131099 \\
\hline GO Biological Processes & GO:0043409 & negative regulation of MAPK cascade & -4.84419894 \\
\hline GO Biological Processes & GO:1903351 & cellular response to dopamine & -4.25433626 \\
\hline GO Biological Processes & GO:0035666 & TRIF-dependent toll-like receptor signaling pathway & -3.72907579 \\
\hline GO Biological Processes & GO:0060398 & regulation of growth hormone receptor signaling pathway & -3.59181535 \\
\hline GO Biological Processes & GO:1904407 & positive regulation of nitric oxide metabolic process & -3.51272839 \\
\hline GO Biological Processes & GO:0009190 & cyclic nucleotide biosynthetic process & -3.38699864 \\
\hline GO Biological Processes & GO:0000226 & microtubule cytoskeleton organization & -3.37279545 \\
\hline GO Biological Processes & GO:0030258 & lipid modification & -3.34130208 \\
\hline GO Biological Processes & GO:0016569 & covalent chromatin modification & -3.19790154 \\
\hline GO Biological Processes & GO:0046856 & phosphatidylinositol dephosphorylation & -3.11446184 \\
\hline GO Biological Processes & GO:0046777 & protein autophosphorylation & -3.11274876 \\
\hline GO Biological Processes & GO:0090336 & positive regulation of brown fat cell differentiation & -3.07693529 \\
\hline GO Biological Processes & GO:0060391 & positive regulation of SMAD protein signal transduction & -3.07693529 \\
\hline GO Biological Processes & GO:0007099 & centriole replication & -3.06412444 \\
\hline GO Biological Processes & GO:0031570 & DNA integrity checkpoint & -2.93592311 \\
\hline GO Biological Processes & GO:0019835 & cytolysis & -2.87954652 \\
\hline GO Biological Processes & GO:0007030 & Golgi organization & -2.79292724 \\
\hline GO Biological Processes & GO:0048539 & bone marrow development & -2.684576 \\
\hline KEGG Pathway & hsa00310 & Lysine degradation & -5 \\
\hline KEGG Pathway & hsa04668 & TNF signaling pathway & -3.4 \\
\hline
\end{tabular}




\begin{tabular}{lll}
\hline Category & Term & Description \\
\hline KEGG Pathway & hsa04621 & NOD-like receptor signaling pathway \\
KEGG Pathway & hsa05020 & Prion diseases \\
KEGG Pathway & hsa00514 & Other types of O-glycan biosynthesis \\
KEGG Pathway & hsa04140 & Autophagy - animal \\
KEGG Pathway & hsa04912 & GnRH signaling pathway \\
KEGG Pathway & hsa04070 & Phosphatidylinositol signaling system \\
KEGG Pathway & hsa04210 & Apoptosis \\
KEGG Pathway & hsa04550 & Signaling pathways regulating pluripotency of stem cells \\
\hline
\end{tabular}

Table 3. The common hub genes of WGCNA and PPI analysis in the blue and magenta modules

\begin{tabular}{|c|c|c|c|c|c|c|c|c|c|c|}
\hline \multirow{2}{*}{$\begin{array}{l}\text { Gene } \\
\text { Symbol }\end{array}$} & \multirow[t]{2}{*}{ Gene Description } & \multirow[t]{2}{*}{ Module } & \multicolumn{4}{|l|}{ WGCNA } & \multirow{2}{*}{$\begin{array}{l}\text { PPI Analysis } \\
\text { K Score }\end{array}$} & \multicolumn{3}{|c|}{ Limma Analysis } \\
\hline & & & GS & GS P value & MM & MM, P value & & Log2FC & FDR & Up or Down \\
\hline ACTG2 & $\begin{array}{l}\text { actin, gamma 2, smooth } \\
\text { muscle, enteric }\end{array}$ & blue & 0.922139646 & 7.51E-09 & 0.923366176 & $6.54 \mathrm{E}-09$ & 5.667 & 3.548158459 & $1.02 \mathrm{E}-08$ & up \\
\hline C1R & $\begin{array}{l}\text { complement } \mathrm{C} 1 \mathrm{r} \\
\text { subcomponent }\end{array}$ & blue & 0.897984733 & 7.81E-08 & 0.96174151 & $1.45 \mathrm{E}-11$ & 4 & 2.208552292 & $1.14 \mathrm{E}-07$ & up \\
\hline $\mathrm{CDH} 3$ & cadherin 3 & blue & 0.931420936 & $2.48 \mathrm{E}-09$ & 0.971836642 & $9.56 \mathrm{E}-13$ & 6.2 & 2.775338929 & 3.97E-09 & up \\
\hline CLU & clusterin & blue & 0.863575842 & $9.38 \mathrm{E}-07$ & 0.878310934 & $3.54 \mathrm{E}-07$ & 4 & 2.044619742 & $1.29 \mathrm{E}-06$ & up \\
\hline COL8A1 & $\begin{array}{l}\text { collagen type VIII alpha } 1 \\
\text { chain }\end{array}$ & blue & 0.92193141 & 7.69E-09 & 0.941172973 & 6.47E-10 & 3 & 4.329881143 & $1.01 \mathrm{E}-08$ & Up \\
\hline GATA6 & GATA binding protein 6 & blue & 0.98763453 & $6.14 \mathrm{E}-16$ & 0.972736196 & $7.16 \mathrm{E}-13$ & 5.667 & 4.878194698 & $1.42 \mathrm{E}-15$ & up \\
\hline HOXC6 & homeobox C6 & blue & 0.944317318 & 3.99E-10 & 0.90875176 & $2.98 \mathrm{E}-08$ & 4.8 & 3.487499137 & $6.36 \mathrm{E}-10$ & up \\
\hline LMOD1 & leiomodin 1 & blue & 0.880126801 & 3.12E-07 & 0.947799849 & $2.26 \mathrm{E}-10$ & 5.667 & 2.476035697 & 4.14E-07 & up \\
\hline MYH11 & myosin heavy chain 11 & blue & 0.933072838 & 2.00E-09 & 0.975751992 & $2.52 \mathrm{E}-13$ & 5.667 & 3.708376486 & 2.96E-09 & Up \\
\hline MYLK & myosin light chain kinase & blue & 0.914954646 & $1.62 \mathrm{E}-08$ & 0.94723591 & $2.48 \mathrm{E}-10$ & 5.667 & 2.125367821 & 2.54E-08 & up \\
\hline MYOCD & myocardin & blue & 0.859995861 & 1.17E-06 & 0.929712545 & 3.08E-09 & 5.667 & 3.441041169 & 1.43E-06 & up \\
\hline PROS1 & protein S (alpha) & blue & 0.934607399 & 1.64E-09 & 0.984275338 & $5.27 \mathrm{E}-15$ & 4 & 2.899254277 & 2.67E-09 & up \\
\hline SERPING1 & serpin family $\mathrm{G}$ member 1 & blue & 0.859067114 & $1.23 \mathrm{E}-06$ & 0.937390849 & 1.12E-09 & 4 & 2.424791429 & 1.61E-06 & up \\
\hline TAGLN & transgelin & blue & 0.909061849 & 2.89E-08 & 0.959903156 & $2.20 \mathrm{E}-11$ & 5.667 & 2.815676431 & 3.97E-08 & up \\
\hline THBS1 & thrombospondin 1 & blue & 0.764480323 & $8.68 \mathrm{E}-05$ & 0.84490284 & 2.77E-06 & 4 & 2.047575043 & 0.000118406 & up \\
\hline THBS2 & thrombospondin 2 & blue & 0.870101501 & $6.18 \mathrm{E}-07$ & 0.92128971 & $8.25 \mathrm{E}-09$ & 3 & 2.397111323 & 8.17E-07 & up \\
\hline $\mathrm{ABCC} 8$ & $\begin{array}{l}\text { ATP binding cassette } \\
\text { subfamily } \mathrm{C} \text { member } 8\end{array}$ & magenta & 0.726050115 & 0.000966995 & 0.922624053 & $1.35214 \mathrm{E}-07$ & 3 & 2.368106299 & 0.000000474 & Up \\
\hline COL4A6 & $\begin{array}{l}\text { collagen type IV alpha } 6 \\
\text { chain }\end{array}$ & magenta & 0.831303264 & 3.54879E-05 & 0.873736048 & 4.59798E-06 & 4.71 & 3.592049292 & $5.82 \mathrm{E}-08$ & up \\
\hline COL5A3 & $\begin{array}{l}\text { collagen type } \mathrm{V} \text { alpha } 3 \\
\text { chain }\end{array}$ & magenta & 0.864722139 & 7.50448E-06 & 0.96496314 & 4.02139E-10 & 4.71 & 2.690812909 & $6.65 \mathrm{E}-08$ & up \\
\hline CXCL13 & $\begin{array}{l}\text { C-X-C motif chemokine } \\
\text { ligand } 13\end{array}$ & magenta & 0.676038594 & $2.89 \mathrm{E}-03$ & 0.808787134 & 8.47082E-05 & 20.9 & 2.692421845 & 0.00000669 & up \\
\hline CYP2E1 & $\begin{array}{l}\text { cytochrome P450 family } 2 \\
\text { subfamily E member } 1\end{array}$ & magenta & 0.813393515 & 7.1566E-05 & 0.937472578 & 2.85784E-08 & 4.5 & 2.3219305 & $4.78 \mathrm{E}-08$ & up \\
\hline CYP4B1 & $\begin{array}{l}\text { cytochrome P450 family } 4 \\
\text { subfamily B member } 1\end{array}$ & magenta & 0.736111943 & 0.000754364 & 0.858320627 & 1.04106E-05 & 4.5 & 3.0752333 & 0.000000839 & up \\
\hline DKK1 & $\begin{array}{l}\text { dickkopf WNT signaling } \\
\text { pathway inhibitor } 1\end{array}$ & magenta & 0.697058489 & 0.001871854 & 0.815860925 & $6.53 \mathrm{E}-05$ & 4.71 & 3.533265993 & 0.000000546 & up \\
\hline FSTL3 & follistatin like 3 & magenta & 0.76382863 & 0.000358509 & 0.894831724 & $1.24 \mathrm{E}-06$ & 5.067 & 2.997977144 & 0.000000244 & up \\
\hline KLF4 & Kruppel like factor 4 & magenta & 0.725261009 & 0.000985574 & 0.884368596 & $2.45 \mathrm{E}-06$ & 4.71 & 3.223838581 & $9.88 \mathrm{E}-08$ & up \\
\hline NR4A2 & $\begin{array}{l}\text { nuclear receptor subfamily } \\
4 \text { group A member } 2\end{array}$ & magenta & 0.800101777 & 0.000115047 & 0.864686086 & 7.52E-06 & 4.71 & 3.44866934 & $4.88 \mathrm{E}-08$ & up \\
\hline PROK1 & prokineticin 1 & magenta & 0.830706844 & 3.6373E-05 & 0.807977649 & $8.72 \mathrm{E}-05$ & 11 & 4.082022638 & $1.93 \mathrm{E}-08$ & up \\
\hline WDR27 & WD repeat domain 27 & magenta & 0.790440633 & 0.000159037 & 0.950669894 & $5.02 \mathrm{E}-09$ & 3 & 2.232448342 & 9.99E-08 & up \\
\hline
\end{tabular}

WGCNA: weight gene co-expression network analysis, PPI: protein-protein interaction, GS: gene significance, MM: module membership, log 2 FC: log2 (Fold-Change) values of differentially expressed genes.

\section{Validation of hub genes}

Independent datasets were used to identify the hub genes. We compared the expression of each hub gene in endometriosis. Fifteen hub genes were differentially expressed between the EC and Ctrl in GSE 7305 (Figure 8A), and seven hub genes were differentially expressed between the EU and Ctrl in GSE 51981 (Figure 8B). Boxplots were used to show the validation results (Figure 8A-B).

\section{Discussion}

Endometriosis is a non-malignant gynecological disease whose pathogenesis is still unclear. The absence of biomarkers may contribute to the long delay between disease onset and diagnosis. Hence, it is imperative to identify novel molecular biomarkers that may enable early diagnosis and personalized treatment. For the first time, our study identified endometriosis-associated hub genes using WGCNA, which may hold important clues regarding the pathogenesis of endometriosis, provide valuable resources for the identification of endometriosis biomarkers and thus may improve the clinical management of this disease. 
A

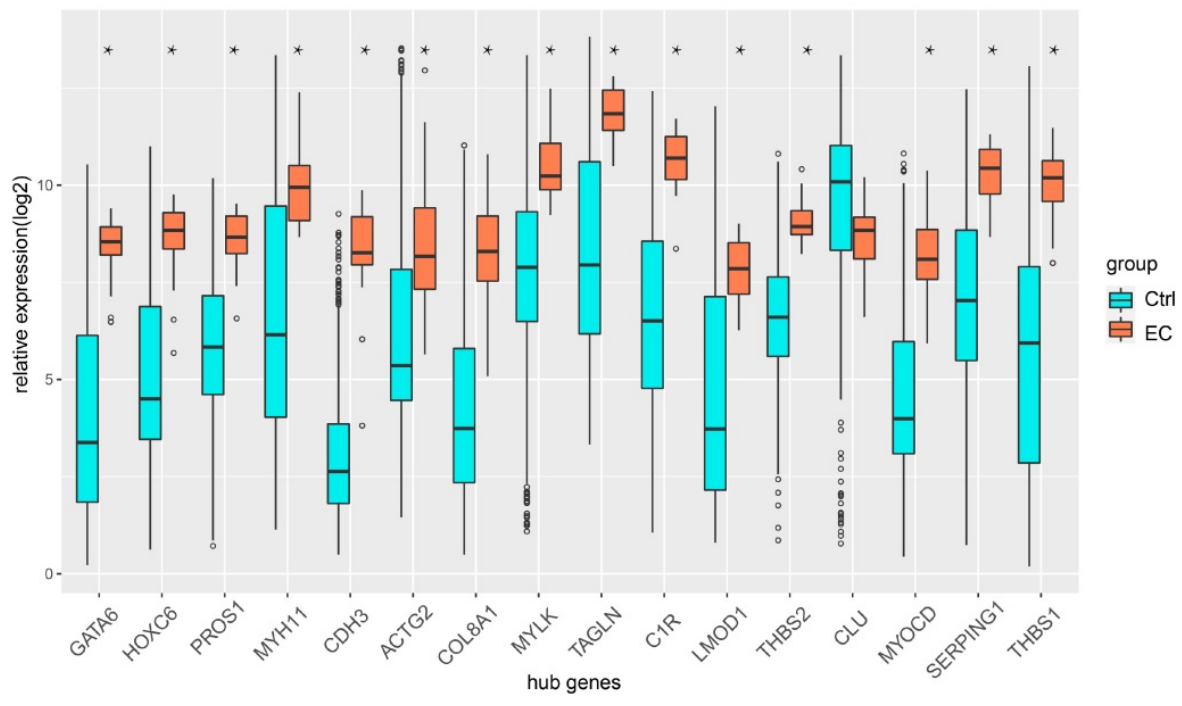

B

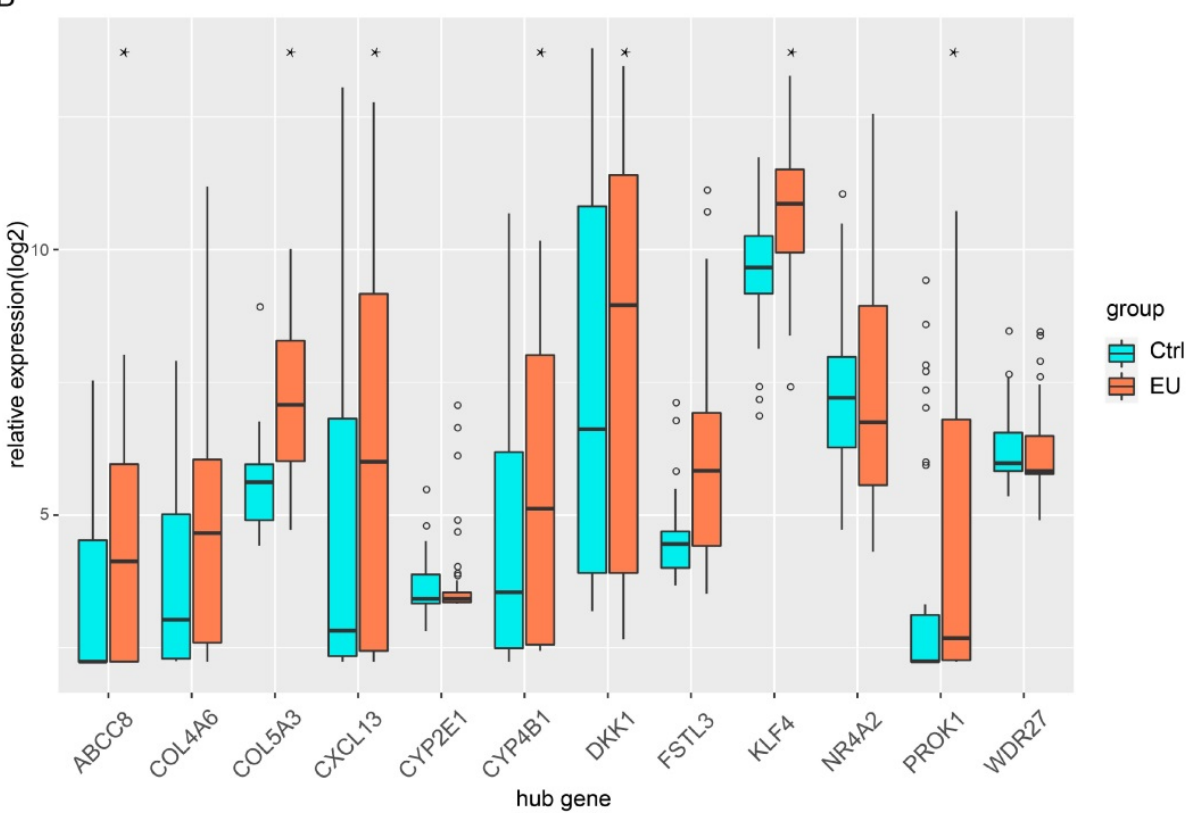

Figure 8. Validation of hub genes in the independent GEO datasets. (A) Boxplot shows the hub gene expression in EC and Ctrl. (B) Boxplot shows the hub gene expression in EU and Ctrl ( ${ }^{p} p<0.05$ versus $C$ trrl).

WGCNA can produce more robust results compared with other bioinformatics methods $[17,18]$ because it constructs weighted co-expression networks based on the similarities of gene expression profiles and focuses on the correlation between the co-expressed modules and clinical traits. Hub genes are defined as the highly connected nodes that contribute to a phenotype or disease [19]. Therefore, this method has been used to identify biologically relevant modules and biomarkers in different diseases [20]. Endometriosis is a benign disease, although, similar to cancer, it has characteristics of being invasive and migratory. In our study, EC and EUassociated hub modules were identified. Function enrichment analyses showed that the genes in the blue and magenta modules had different roles and both were significantly associated with endometriosis, which demonstrated our analysis. For example, genes in the EC-associated blue module played roles in autophagy, focal adhesion (the initiation step for disease progression [21]) and cancer, all of which were involved in the pathogenesis of endometriosis $[22,23]$. Previous studies showed that S100A7 promoted the development of endometriosis by activating NF-kappaB signaling pathway [24]. In our study, the genes in EU-associated magenta module played roles in the regulation of growth hormone receptor signaling pathway, NF-kappaB signaling and 
GnRH signaling pathway, which induced an estrogen-rich and inflammatory microenvironment involved in cell division, cell movement and survival in endometriosis [20,25-27]. As a result, we assume that hormone receptor signaling or inflammatory microenvironment may promote the passing of EU through oviducts and migrating to the ovarian surfaces, and adhesion and autophagy correlated genes may induce the successful ectopic implantation of endometrium (EC) and formation of endometriotic lesions. Taken together, dysregulated genes in the EU may be responsible for the increased propensity of endometrial debris ectopic implantation and for early events that lead to the establishment of lesions. Dysregulated genes in the EC may contribute to the lesion formation and influence the progression of the disease.

To better understand the pathogenesis of endometriosis, WGCNA and PPI analyses were used to identify the EC and EU-associated hub genes. Some hub mRNAs, such as TAS2R3, TAS2R41, SERPING1, CASR, CCKAR, GPR55, HCRTR2, CRH, HTR5A, CFTR, and ENAM, were also key enriched genes in the GSEA. For instance, SERPING1 was involved in the complement and coagulation cascades, both NR4A2 and ABCC8 played important roles in $\mathrm{ABC}$ transporters, and CYP2E1 was involved in the pathway of steroid hormone biosynthesis. In addition, some identified hub genes of the EC (TAGLN, GATA6, CDH3, CLU, COL8A1, MYH11, MYOCD) and EU (CXCL13, DDK-1, KLF4, CYP2E1, CYP4B1 and PROK1) have been reported be associated with endometriosis. For example, TAGLN may be involved in cell invasion, migration, and differentiation in endometriosis [28]. GATA6 is an essential gene in the activation of estrogen synthesis and may become a molecular marker in endometriotic lesions [29,30]. Endometrial CXCL13 expression may play an important role in the pathophysiology of endometriosis [31]. MiR-200b inhibited invasive growth in endometriosis by targeting KLF4 [32]. Dysregulated endometrial PROK1 expression may be correlated with the progesterone resistance of endometriosis [33]. Most importantly, we discovered some novel and important genes, including HOXC6, PROS1, SERPING1, MYLK, ACTG2 and THBS2 in the ectopic endometrium, and NR4A2, ABCC8, COL4A6, COL5A3, FSTL3 and WDR27 in the eutopic endometrium. For example, HOXC6 was found to regulate the response to hormonal signals, and the overexpression of FSTL3 significantly improved angiogenesis and neovascularization in the induced pluripotent stem cells [34]. These hub genes may provide new mechanisms for endometriosis and will be investigated in the future.
Our study identified multi-molecule biomarkers in endometriosis. However, some patients of the validation datasets had incomplete clinical information, which affected further data exploration. The identified genes will be further validated by clinical specimens and in vitro experiments for their application in endometriosis.

\section{Conclusion}

Our study for the first time analyzed the gene expression files of the eutopic and ectopic endometrium in women with endometriosis using WGCNA, explored the distinct functions of the eutopic and ectopic endometrium, and identified co-expression modules and potential biomarkers for endometriosis. Our study may improve the understanding of the pathogenesis of endometriosis and provide references for endometriosis-associated biomarkers and therapeutic targets.

\section{Abbreviations}

WGCNA: Weighted gene co-expression network analysis; GEO: gene expression omnibus; DEGs: Differentially expressed genes; PPI: Protein-protein interaction; Limma: linear models for microarray data; MCODE: Molecular Complex Detection; TOM: Topological overlap matrix; GO: Gene Ontology; KEGG: Kyoto Encyclopedia of Genes and Genomes; GSEA: Gene-set enrichment analysis; GS: gene significance; MM: module membership; STRING: The search tool for retrieval of interacting genes.

\section{Acknowledgments}

\section{Data Availability}

The following publicly-available datasets were analyzed in this study: [https://www.ncbi.nlm.nih. gov/geo/query/acc.cgi?acc=GSE120103]; [https:// www.ncbi.nlm.nih.gov/geo/query / acc.cgi?acc $=\mathrm{GSE}$ 7305]; [https://www.ncbi.nlm.nih.gov/geo/query/ acc.cgi]; [https://www.ncbi.nlm.nih.gov/geo/ query / acc.cgi?acc=GSE51981].

\section{Authors' Contribution}

J.W., X.F. and X.X. conceived and designed the study. J.W., X.F. analyzed the data. J.W. wrote the manuscript. X.F. and X.X. critically evaluated the data and contributed to the writing of the manuscript.

\section{Funding}

This work was supported by the National Natural Science Foundation of China [grant number 81671437, 81771558], Natural Science Foundation of Hunan Province [grant number 2020JJ4814], the Fundamental Research Funds for the Central 


\section{Universities of Central South University [grant number 2020zzts285].}

\section{Competing Interests}

The authors have declared that no competing interest exists.

\section{References}

1. Zondervan KT, Becker CM, Koga K, et al. Endometriosis. Nat Rev Dis Primer 2018; 4: 1-25.

2. Giudice LC, Kao LC. Endometriosis. The Lancet 2004; 364: 1789-1799.

3. McKinnon B, Mueller M, Montgomery G. Progesterone Resistance in Endometriosis: an Acquired Property? Trends Endocrinol Metab 2018; 29: 535 548 .

4. Zhang Z, Liu R, Jin R, et al. Integrating Clinical and Genetic Analysis of Perineural Invasion in Head and Neck Squamous Cell Carcinoma. Front Oncol 2019; 9: 434.

5. Tang J, Kong D, Cui Q, et al. Prognostic Genes of Breast Cancer Identified by Gene Co-expression Network Analysis. Front Oncol 2018; 8: 374.

6. Yin L, Cai Z, Zhu B, et al. Identification of Key Pathways and Genes in the Dynamic Progression of HCC Based on WGCNA. Genes 2018; 9:92.

7. Hever A, Roth RB, Hevezi P, et al. Human endometriosis is associated with plasma cells and overexpression of B lymphocyte stimulator. Proc Natl Acad Sci U S A 2007; 104: 12451-12456.

8. Bhat MA, Sharma JB, Roy KK, et al. Genomic evidence of $Y$ chromosome microchimerism in the endometrium during endometriosis and in cases of infertility. Reprod Biol Endocrinol RBE 2019; 17: 22

9. Ritchie ME Silver J, Oshlack A, et al. A comparison of background correction methods for two-colour microarrays. Bioinforma Oxf Engl 2007; 23: 2700-2707.

10. Ritchie ME, Phipson $\mathrm{B}, \mathrm{Wu} \mathrm{D}$, et al. limma powers differential expression analyses for RNA-sequencing and microarray studies. Nucleic Acids Res 2015; 43: e47.

11. Ritchie ME, Phipson B, Wu D, et al. limma powers differential expression analyses for RNA-sequencing and microarray studies. Nucleic Acids Res 2015; 43: e47.

12. Magbanua MJM, Richman EL, Sosa EV, et al. Physical activity and prostate gene expression in men with low-risk prostate cancer. Cancer Causes Control 2014; 25: 515-523.

13. Bader GD, Hogue CWV. An automated method for finding molecular complexes in large protein interaction networks. BMC Bioinformatics 2003; 4: 2.

14. Langfelder P, Horvath S. WGCNA: an R package for weighted correlation network analysis. BMC Bioinformatics 2008; 9: 559 .

15. Li A, Horvath S. Network neighborhood analysis with the multi-node topological overlap measure. Bioinformatics 2007; 23: 222-231.

16. Botía JA, Vandrovcova J, Forabosco P, et al. An additional k-means clustering step improves the biological features of WGCNA gene co-expression networks. BMC Syst Biol 2017; 11: 47.

17. Zhang B, Horvath $\mathrm{S}$. A general framework for weighted gene co-expression network analysis. Stat Appl Genet Mol Biol 2005; 4: Article17.

18. Li J, Zhou D, Qiu W, et al. Application of Weighted Gene Co-expression Network Analysis for Data from Paired Design. Sci Rep 2018; 8: 622

19. Bakhtiarizadeh MR, Hosseinpour B, Shahhoseini M, et al. Weighted Gene Co-expression Network Analysis of Endometriosis and Identification of Functional Modules Associated With Its Main Hallmarks. Front Genet 2018; 9: 453.

20. Gentilini D, Busacca M, Di Francesco S, et al. PI3K/Akt and ERK1/2 signalling pathways are involved in endometrial cell migration induced by 17betaestradiol and growth factors. Mol Hum Reprod 2007; 13: 317-322.

21. Tsai H-W, Huang M-T, Wang P-H, et al. Decoy receptor 3 promotes cell adhesion and enhances endometriosis development: DcR3 promotes cell adhesion and enhances endometriosis development. J Pathol 2018; 244: 189202.

22. Kuessel L, Wenzl R, Proestling K, et al. Soluble VCAM-1/soluble ICAM-1 ratio is a promising biomarker for diagnosing endometriosis. Hum Reprod Oxf Engl 2017; 32: 770-779.

23. Zhan L, Yao S, Sun S, et al. NLRC5 and autophagy combined as possible predictors in patients with endometriosis. Fertil Steril 2018; 110: 949-956.

24. Sun Q, Cao Y, Lan Y, et al. S100A7 promotes the development of human endometriosis by activating NF-kB signaling pathway in endometrial stromal cells. Cell Biol Int 2021; 45: 1327-1335.

25. Chowdhury I, Banerjee S, Driss A, et al. Curcumin attenuates proangiogenic and proinflammatory factors in human eutopic endometrial stromal cells through the NF-kB signaling pathway. J Cell Physiol 2019; 234: 6298-6312.

26. González-Ramos R, Defrère S, Devoto L. Nuclear factor-kappaB: a main regulator of inflammation and cell survival in endometriosis pathophysiology. Fertil Steril 2012; 98: 520-528.

27. Uimari O, Rahmioglu N, Nyholt DR, et al. Genome-wide genetic analyses highlight mitogen-activated protein kinase (MAPK) signaling in the pathogenesis of endometriosis. Hum Reprod Oxf Engl 2017; 32: 780-793.
28. Dos Santos Hidalgo G, Meola J, Rosa E Silva JC, et al. TAGLN expression is deregulated in endometriosis and may be involved in cell invasion, migration, and differentiation. Fertil Steril 2011; 96: 700-703.

29. Bernardi LiaA, Dyson MT, Tokunaga H, et al. The Essential Role of GATA6 in the Activation of Estrogen Synthesis in Endometriosis. Reprod Sci 2019; 26: 60-69.

30. Izawa M, Taniguchi F, Harada T. GATA6 expression promoted by an active enhancer may become a molecular marker in endometriosis lesions. Am J Reprod Immunol 2019; 81: e13078.

31. Franasiak JM, Burns KA, Slayden O, et al. Endometrial CXCL13 Expression is Cycle Regulated in Humans and Aberrantly Expressed in Humans and Rhesus Macaques with Endometriosis. Reprod Sci 2015; 22: 442-451.

32. Eggers JC, Martino V, Reinbold R, et al. microRNA miR-200b affects proliferation, invasiveness and stemness of endometriotic cells by targeting ZEB1, ZEB2 and KLF4. Reprod Biomed Online 2016; 32: 434-445.

33. Tiberi F, Tropea A, Apa R, et al. Prokineticin 1 mRNA expression in the endometrium of healthy women and in the eutopic endometrium of women with endometriosis. Fertil Steril 2010; 93: 2145-2149.

34. Ansari KI, Hussain I, Shrestha B, et al. HOXC6 is transcriptionally regulated via coordination of MLL histone methylase and estrogen receptor in an estrogen environment. J Mol Biol 2011; 411: 334-349. 